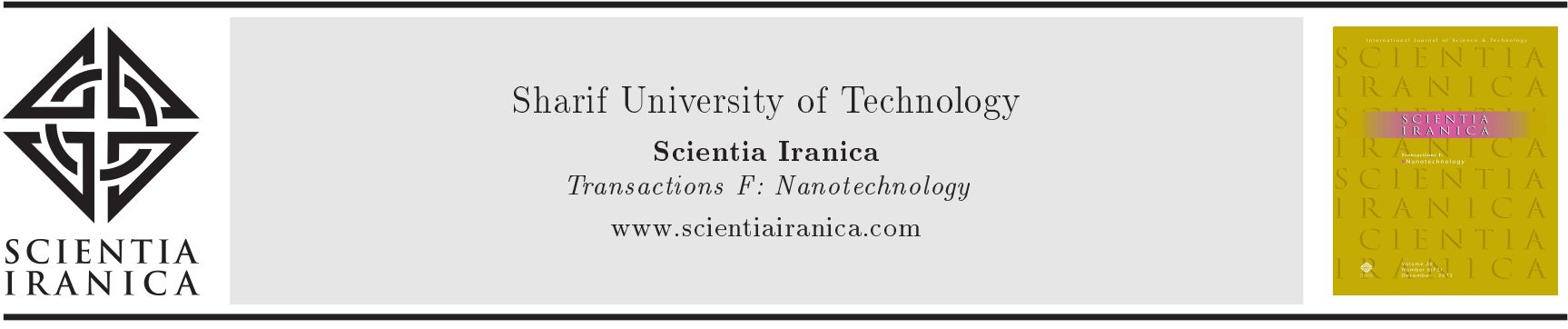

\title{
Output power enhancement of Rhodamine 6G dye laser by colloidal gold nanoparticles
}

\author{
A. Moshaii* \\ Department of Physics, Tarbiat Modares University, Tehran, P.O. Box 14115-175, Iran.
}

Received 10 October 2015; received in revised form 9 July 2016; accepted 8 November 2016

\section{KEYWORDS}

Gold colloidal

nanoparticles;

Rh6G dye laser;

Plasmonic;

Power enhancement.

\begin{abstract}
Improvement of the efficiency of a Rhodamine 6G dye laser by adding proper concentrations of gold colloidal nanoparticles into the dye solution has experimentally been investigated. Gold colloidal nanoparticles, due to their strong plasmonic peak in the visible region, are expected to have a considerable interaction with Rhodamine $6 \mathrm{G}$ dye molecules with broad emission spectrum in the visible range. It is shown that with addition of enough low concentrations (less than $0.4 \mu \mathrm{M} / \mathrm{l}$ ) of gold nanoparticles to the dye solution, both the output power and the efficiency of the dye laser increase. The best improvement of the laser efficiency reaches $15 \%$. Two amplifying mechanisms exist for efficiency improvement by the nanoparticles, which are resonant energy transfer from the plasmonic nanoparticles to the dye molecules and the high near field around the nanoparticles. The results indicate that in low concentrations of gold nanoparticles, the amplifying mechanisms dominate the dissipating mechanism of scattering of the pumped light by the nanoparticles. This leads to the efficiency improvement by addition of the nanoparticles.
\end{abstract}

(C) 2016 Sharif University of Technology. All rights reserved.

\section{Introduction}

Dye lasers, due to capability to produce tunable laser lights in a wide range of wavelengths in addition to producing $\mathrm{CW}$ and pulsed lasers, have many applications in science [1-6], medicine [7-11], and industry [1214]. A dye laser is generally pumped by a flash lamp or by the light of another laser. Usually, the efficiency of dye lasers is low, typically less than $5 \%$ [15]. Therefore, increasing the efficiency and output power of dye lasers is one offavorite challenges for the researchers in the field. The main reason for the low efficiency of dye lasers is existence of some nonradiative transitions in the dye molecules, including the intersystem crossing processes from a singlet excited state to lower triplet states $[16,17]$. This generally leads to reduction of the population inversion in the excited

* Fax: +982182883459

E-mail address: Moshaii@modares.ac.ir state, which consequently diminishes the number of lasing transitions [18].

Usually, by increasing the pumping power and the concentration of dye molecules, it is possible to produce higher output power from a typical dye laser. However, enough pumping power leads to cell damaging in which both the material inside the cell and the cell window are damaged [19]. Also, increasing the dye concentration results in distortion of the output spatial profile [15]. Therefore, for moderate dye concentrations, finding a method for increasing the excitation rate without reaching the threshold of cell burning is a desirable goal in developing the dye lasers. The main idea of this paper is to use the enhanced electromagnetic field of localized surface plasmons as a promising method for this purpose.

Localized Surface Plasmon Resonance (LSPR) is produced by collective oscillations of conduction electrons of metal nanoparticles due to interaction with an external field. The resonance occurs when the 
frequency of the electromagnetic field is adjusted to the natural frequency of oscillations of electrons in the metal nanoparticle. This resonance depends on the size [20,21] and the shape [22] of the nanoparticles in addition to the dielectric constants of the surrounding medium [23]. Around the plasmonic nanoparticles, in the near field regions, the electric field can be much greater than the incident field $[24,25]$. Such enhanced electric field can be used for improving the efficiency of a dye laser. The enhanced localized electromagnetic field near the metal nanoparticles can facilitate the excitation of a molecule or a quantum dot [26]. In fact, the excitation rate of the molecule close to a nanoparticle is proportional to the electric field at the location of the molecule, which can increase by the near field enhancement of LSPR [27]. In addition, both radiative and non-radiative decay rates of an excited molecule can change by presence of a metal nanoparticle around the excited molecule.

The interaction of metal nanoparticles with dye molecules has been studied by several researchers [2837]. Both fluorescence [38-42] and fluorescence enhancement of dye molecules [43-45] have been reported as the results of the nanoparticle-dye interaction. One of the notable reports in the field is the enhancement of spontaneous emission of Rh6G molecules (up to 45\%) by addition of aggregated silver nanoparticles to the dye solution [46]. When a dye molecule is placed in the vicinity of a metal nanoparticle, two mechanisms can amplify the excitation of the dye molecule. The first is a resonant energy transfer from the plasmonic nanoparticle to the dye molecule, which can excite the dye molecule to an upper level [47]. Of course, the rate of energy transfer from the donor particle to the acceptor molecule depends on the spectral overlap of the donor's emission spectrum and the acceptor's absorption spectrum. The second mechanism is the higher electric field that the dye molecule senses in the near field region of the nanoparticle [48]. In addition, placing the nanoparticles close to the dye molecules can conduct some dissipating processes that non-radiatively bring the excited dye molecule to some lower levels and this reduces the emission of the dye molecule.

In this paper, we study improvement of the output power of an Rh6G dye laser by incorporating the plasmonic properties of gold nanoparticles in the framework of a dye circulating system. A Littrow configuration has been used in the optical resonator to achieve a tuned and stable wavelength profile with less than $1 \mathrm{~nm}$ spectral width. This system has the merit of long time lasing stability (even with the nanoparticles inside the dye solution) in addition to lack of any photo-bleaching problem of the active media. After synthesizing the gold nanoparticles and mixing them with a fixed dye solution, we measured the effect of various concentrations of gold nanoparticles on the improvement of output power of the laser. The results indicate that under the optimum concentration of gold nanoparticles, it is possible to increase the laser efficiency by about $15 \%$.

\section{Experimental setup}

We measured the output power of an oscillator dye laser with different mixtures of Rh6G dye and colloidal gold nanoparticles both dissolved in ethanol. The concentration of dye in the solutions was fixed at $0.01 \mathrm{mM} / 1$. The experimental setup for measuring the output power of the dye laser has schematically been shown in Figure 1. The active media was a rectangular cubic dye cell with the dimensions of $1 \times$ $1 \mathrm{~mm}^{2}$ and the length of $20 \mathrm{~mm}$. The dye cell was transversely pumped with the cross sectional area of $1 \times 20 \mathrm{~mm}^{2}$. The dye solution was circulated in the cell, with the flow of $2 \mathrm{l} / \mathrm{min}$. The pumping system was a pulsed Q-switched Nd:YAG laser (second harmonic $\lambda=532 \mathrm{~nm}$ ), with the high repetition rate of $10 \mathrm{kHz}$ and the pulse duration of $10 \mathrm{~ns}$. The intensities of two polarizations of the light of the pump laser reaching the dye cavity can be controlled by a combination of a half-wavelength slab and a polarizer. The powermeters (PM1 and PM2) measured two sample intensities of the perpendicular polarizations of the pump light. A Littrow configuration was used for the resonator of the dye laser with a 1200 line $/ \mathrm{mm}$ grating as the back mirror and an $80 \%$ reflection mirror as the front one. A portion of the output power of the dye laser was measured by the powermeter PM3 after passing through the sampler SA2. Also, the spectrometer shown in the figure determined the frequency spectrum

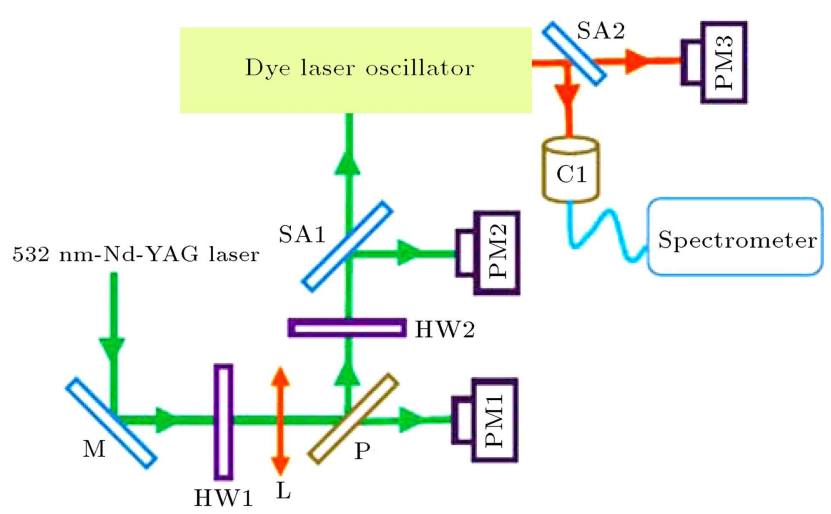

Figure 1. Experimental setup for measuring the output power of an Rh6G dye laser to investigate the effect of different concentrations of gold nanoparticles on the laser performance. Two half-wavelength slabs of HW1 and HW2 in addition to the polarizer $P$ totally control the intensities of two polarizations of the pump light reaching the dye cavity. The samplers SA1 and SA2 provide two samples of the intensities of the pump and the output lights reaching the photomultiplier tubes (PM1 and PM2). 
of the dye laser output. It should be mentioned that all powermeters used in this work were similar and provided from Gentec.

The gold nanoparticles for this work were synthesized by the method of $[27,49]$. The average diameter size of the synthesized spherical nanoparticles was measured by a TEM to be around $10 \mathrm{~nm}$ (Figure 2), which was much greater than the size of Rh6G dye molecules. Figure 3 schematically shows the size of an Rh6G dye molecule in front of a $10 \mathrm{~nm}$ gold nanoparticle. To be sure that the nanoparticles remained monodispersed in all experiments, the solutions of nanoparticles before and after mixing with the dye solution were inside the ultrasonic bath for $10 \mathrm{~min}$. By changing the concentration of the nanoparticles in the dye solution, the average distance between a dye molecule and the nanoparticles changed. This effectively allowed us to investigate influence of the distance between the dye molecules and the nanoparticles on improvement of the excitation of the dye molecules. In the experiments,

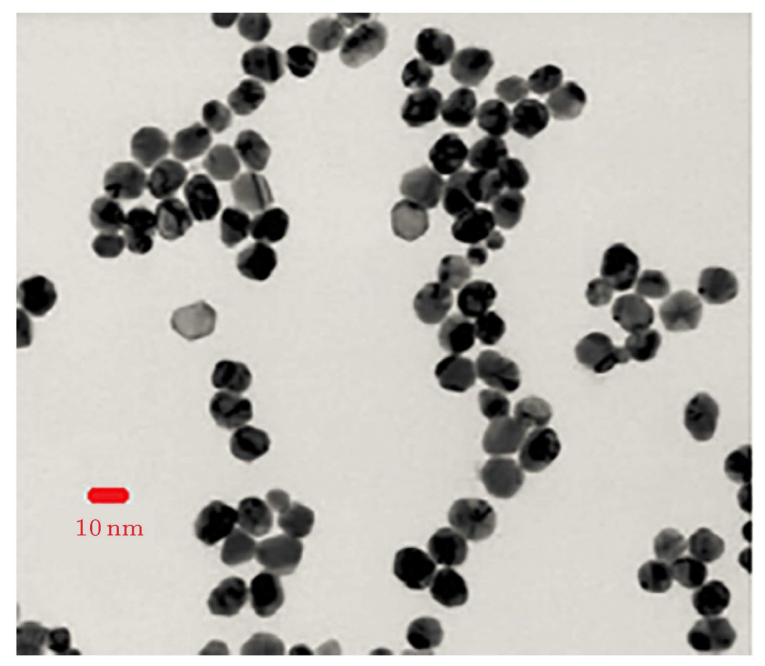

Figure 2. TEM image of the synthesized gold nanoparticles. The image was taken by a Zeiss TEM model EM900 with the energy of $80 \mathrm{keV}$.

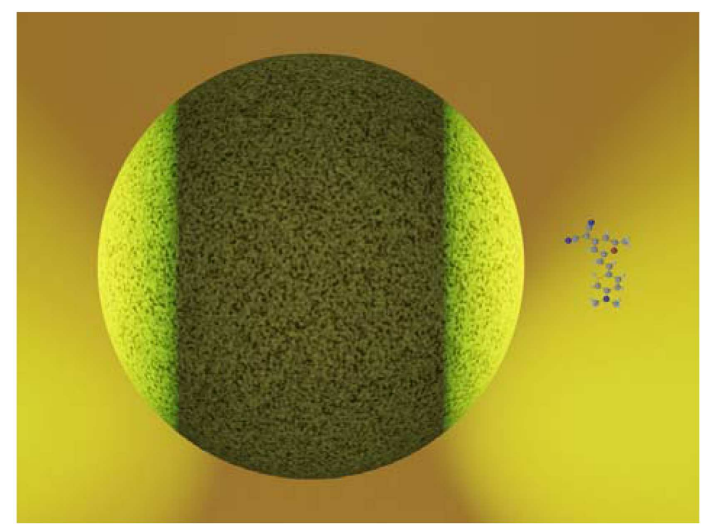

Figure 3. Schematic comparison of the size of an Rh6G dye molecule in front of a $10 \mathrm{~nm}$ gold nanoparticle. we also measured the fluorescence and the absorption spectra of the pure dye solution and the nanoparticles by the setup shown in Figure 4.

\section{Results and discussion}

To investigate the possibility of improvement of the output power of Rh6G dye laser by the gold nanoparticles, we first measured the absorption and the fluorescence spectra of the dye solution in addition to the absorption spectrum of the colloidal gold nanoparticles. The results are shown in Figure 5. It is seen that the plasmonic resonance of the gold nanoparticles has a relatively broad peak around $520 \mathrm{~nm}$. Such peak has a strong tail in the short wavelengths less than $450 \mathrm{~nm}$,

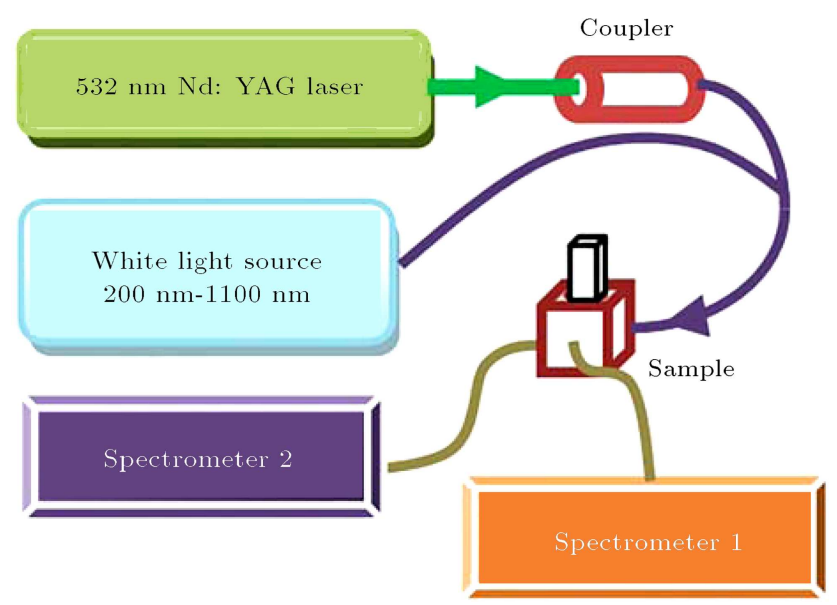

Figure 4. Experimental setup for measuring the fluorescence and theabsorption spectra of the dye solution and the gold nanoparticles. The fluorescence and absorption spectra are obtained by the spectrometers 1 and 2 , respectively.

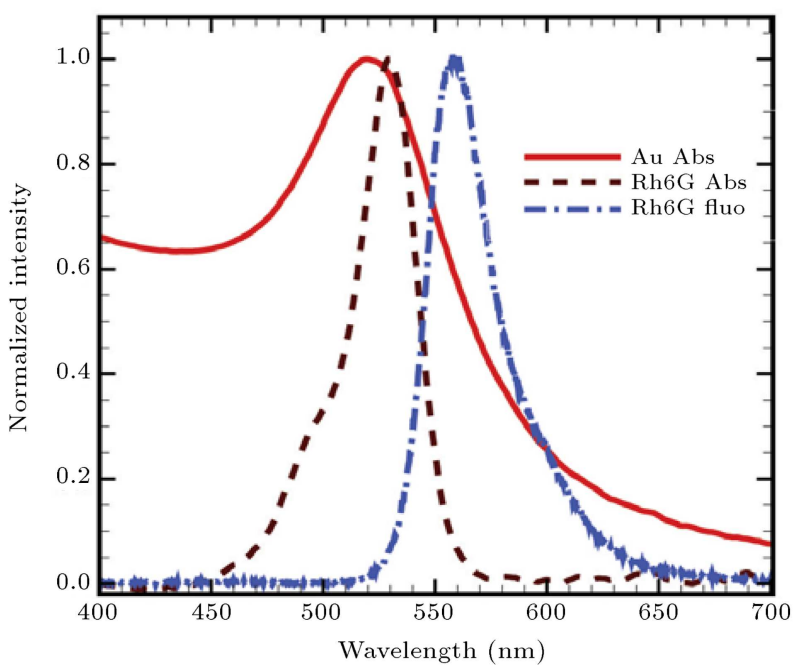

Figure 5. Normalized absorption and fluorescence spectra of Rh6G dye solution in addition to the absorption spectrum of the gold nanoparticles. 
which corresponds to the interband transitions of the gold nanoparticles. On the other hand, the absorption spectrum of the dye solution includes a relatively sharp peak centered at $530 \mathrm{~nm}$. In addition, the fluorescence spectrum of the dye has a sharp peak at $560 \mathrm{~nm}$.

The overlaps between the absorption peak of the gold nanoparticles and the fluorescence and the absorption peaks of the dye molecules are two key factors determining the influences of gold nanoparticles on the radiation properties of the dye molecules. The intersection of the absorption spectra of the nanoparticles and the dye molecules can change the amount excitation of the dye molecules due to resonance energy transfer between the nanoparticles and the dye molecules. Moreover, the overlap between the absorption of nanoparticles and the florescence of the dye molecules leads to change in amount of stimulated emission due to the exchange of energy between the excited dye states and the plasmonic nanoparticles.

As we see in Figure 5, the overlap between the absorption spectra of the nanoparticles and the dye molecules is much greater than the overlap between the fluorescence spectrum of the dye and the absorption spectrum of nanoparticles. Therefore, it is expected that the influence of gold nanoparticles on change in excitation of the dye molecules would become greater than their direct effect on the change of stimulated emission from the excited molecules. If a resonance exchange of energy appears from the nanoparticles to the dye molecules with balance between the plasmon energy and the dye excitation energy, it effectively acts as a pumping amplification and leads to power enhancement of the laser. On the other hand, in the case of transfer of energy from excited dye molecules to the nanoparticles, we will have reduction of excited molecules and decrement in the laser power. In the following, we investigate the experimental conditions in which the presence of the nanoparticles amplifies the lasing transitions of the dye molecules and the conditions in which the presence of nanoparticles diminishes the lasing emission.

By measuring the output power of the dye laser, including different mixtures of Rh6G dye and colloidal $\mathrm{Au}$ nanoparticles, it is possible to investigate the effect of nanoparticles on the laser performance. Figure 6 shows the results in which variations of the laser power versus the change in pumping power are presented for different concentrations of the gold nanoparticles in the dye solutions. It is seen that the trend of increment in the output power versus the pump power is the same for all concentrations of gold nanoparticles. With addition of the nanoparticles to the dye solution, the minimum pump power required for producing a detectable laser light changes. The minimum pump power for initiating the laser light is obtained from the crossing points of the output power curves with the horizontal pump

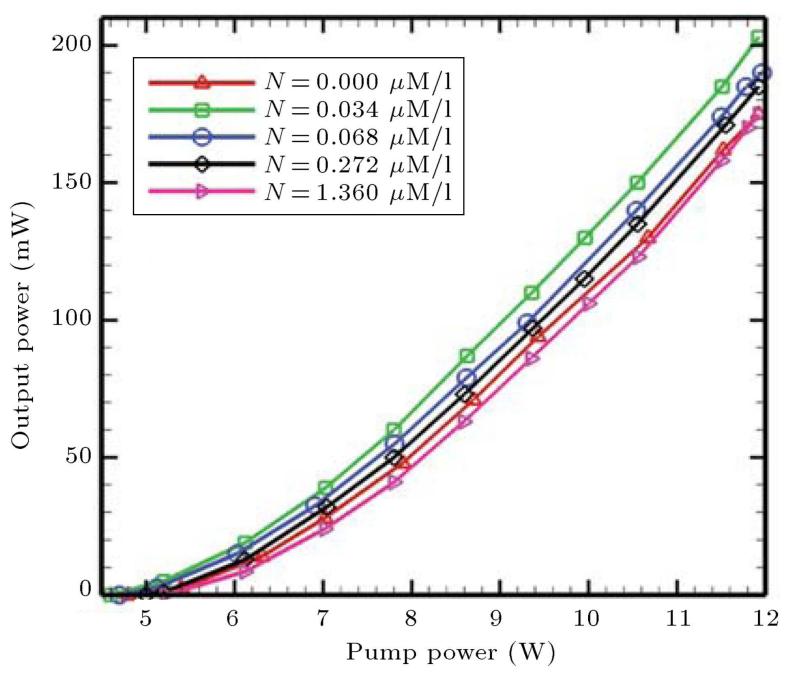

Figure 6. The variations of the output power of the dye laser versus the change in the pumping power for different concentrations of the gold nanoparticles denoted by " $\mathrm{N}$ ".

power axis in Figure 6. The change of minimum pump power means that the efficiency of the laser at the minimum laser output changes with the presence of the nanoparticles in the dye solution.

The results of output power for the laser shown in Figure 6 increase up to the maximum pump power of $12 \mathrm{~W}$. At this pump power, the laser output is in the range of 170 to $205 \mathrm{~mW}$. This pump power is also the maximum pump power for the experimental setup of this work, before the beginning of cell burning, which damages both the dye liquid close to the cell wall and the cell window. This upper threshold depends on the configuration of the cell and is determined by several preliminary tests. We see, between different concentrations of the nanoparticles, the laser output is always maximum for the concentration of $N=$ $0.034 \mu \mathrm{M} / 1$ in all pumping powers.

The results of Figure 7 indicate that for the concentrations of nanoparticles between $0.034 \mu \mathrm{M} / 1$ to $0.272 \mu \mathrm{M} / 1$, with addition of the gold nanoparticles to the dye solution, the output power increases relative to that of pure dye solution. This indicates that in such gold concentrations, the increment in excitation of the dye molecule, which leads to more stimulated (or fluorescence) emission, overwhelms the dissipating processes, including non-radiative energy transfers. The stimulated enhancement is optimized at the gold concentration of $0.034 \mu \mathrm{M} / 1$. However, for enough high gold concentration of $N=1.36 \mu \mathrm{M} / \mathrm{l}$, we see the output power is lower than that of the dye laser without any gold nanoparticles. In this case, the dissipation by the non-radiative energy transfers is dominant in the dyenanoparticle interaction. In fact, the enhancement and quenching of the (stimulated/fluorescence) emission are the main reasons of improvement and degradation of the output power in low and high gold concentrations, 


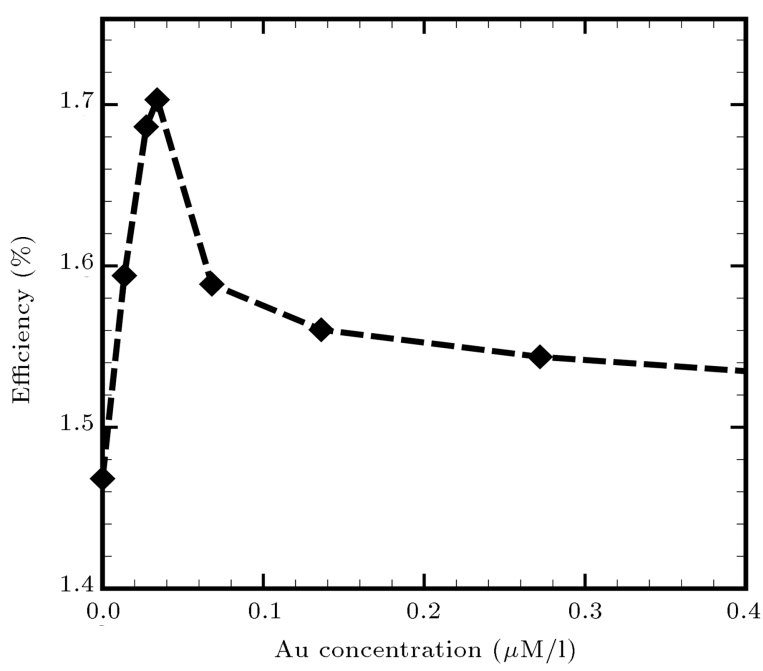

Figure 7. Variations of the efficiency of the dye laser versus the change in the concentration of gold nanoparticles in the dye solution for maximum pump power of 12 watts.

respectively. The results of Figure 7 thoroughly agree with the findings of other researchers in the field [28].

Since $12 \mathrm{~W}$ is the maximum pump power for getting the best output power from the dye laser, we focused on the improvement of the laser efficiency at this pump power. Figure 7 shows variations of the efficiency of the dye laser versus the change in the concentration of gold nanoparticles in the dye solution. It is seen that under the optimum gold concentration, the efficiency increases from $1.43 \%$ to maximum $1.7 \%$, which is about $15 \%$ increment. The best efficiency occurs at the nanoparticles' concentration of $0.034 \mu \mathrm{M} / 1$. Note that in the range of concentrations of gold nanoparticles between 0.1-0.4 $\mu \mathrm{M} / 1$, the efficiency does not remarkably change, although its value is higher than the laser efficiency without using gold nanoparticles. In Figure 8, we have shown the efficiency change of the dye laser with variation of the pump power for the optimum concentration of the nanoparticles; $N=0.034 \mu \mathrm{M} / \mathrm{l}$. Generally, we see that with increment in the pump power, the efficiency of the laser monotonically increases in the dyes both with and without nanoparticles. However, at higher pumping powers, the improvement of the laser output with addition of the gold nanoparticles is more notable.

As mentioned in the previous publications, both fluorescence quenching [41,42] and fluorescence enhancement of dye molecules [38-42] have been reported as the results of interaction between metal nanoparticles and dye molecules. When a dye molecule is placed close to a metal nanoparticle, the excitation of the dye molecule is affected by two exchanges of energy. The first is a resonant energy transfer from the plasmonic nanoparticle to the dye molecule, which can excite the dye molecule to an upper level [47]. The second is

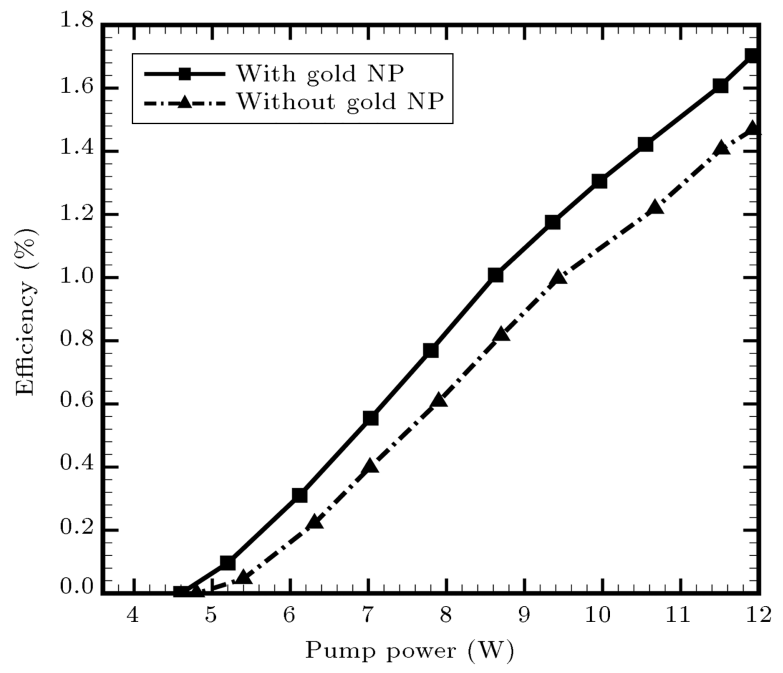

Figure 8. Variations of the Rh6G Dye laser efficiency versus the change in the pump power for two cases of pure dye solution (without gold NP) and dye solution with gold nanoparticles with concentration of $0.034 \mu \mathrm{M} / 1$ (with gold NP).

transfer of energy from the excited dye molecules to the nanoparticles through some dissipating processes that non-radiatively bring the excited dye molecule to some lower levels and this reduces the emission of the dye molecule. Both of these mechanisms can occur and thereby both enhancement and quenching of fluorescence are observed in the experiments.

The experimental results of application of gold nanoparticles for improving the efficiency of an Rh6G dye laser show that in the range of low gold concentrations used in this work, the efficiency always increases with presence of gold nanoparticles in the dye solution. If a dye molecule is placed in the vicinity of a metal nanoparticle, two mechanisms can amplify the excitation of the dye molecule. The first is the high electric field that the dye molecule senses in the near field region of the nanoparticle [26]. The second is the energy transfer from the plasmonic nanoparticle to the dye molecule, transferring the molecule to its excited state [25]. In addition, putting the nanoparticles close to the dye molecules leads to some dissipating processes reducing the excitation of the dye molecule. Two main dissipating processes are the scattering of the pumped light by the nanoparticles, which diminishes the amount of light reaching the dye molecule, and non-radiative energy transfer from the excited dye molecules to the nanoparticles. In fact, the competition between the amplifying processes and the diminishing ones determines the final role of the nanoparticles in improving or declining the dye laser output. The results presented in this work indicate that in the range of low gold concentrations, the two amplifying mechanisms of resonant energy transfer from the plasmonic nanoparticles to the dye molecule and the high near 
field around the nanoparticles dominate the dissipating mechanisms of scattering of the pumped light by the nanoparticles and non-radiative energy transfers from the excited molecules to the nanoparticles.

\section{Conclusion}

In this work, we investigated the influence of gold colloidal nanoparticles on output power of an Rh6G dye laser. Because of strong plasmonic peak of gold nanoperticles in the visible regime (with $\lambda_{\max } \sim 520 \mathrm{~nm}$ ), a remarkable interaction between gold nanoparticles and Rh6G dye molecules can take place. It was shown that in the concentrations less than $0.4 \mu \mathrm{M} / 1$ for the gold nanoparticles, the laser efficiency increases with addition of gold nanoparticles to the dye solution and the optimum improvement of the laser efficiency reaches $15 \%$. This indicates that in these low gold concentrations, the amplifying effects of resonant energy transfer from the plasmonic nanoparticles to the dye molecule in addition to the high near field around the nanoparticles dominate the dissipating effects of scattering of the pumped light by the nanoparticles and non-radiative energy transfers from the dye molecules to the nanoparticles.

\section{Acknowledgments}

This work was supported by Tarbiat Modares University (TMU).

\section{References}

1. Klebniczki, J., Szatmari, S. and Schafer, F.P. "Longitudinally pumped quenched dye laser", J. Phys. E., 21(10), pp. 1005-1006 (1988).

2. Duarte, F.J., Kelley, P., Hillman, L.W. and Liao, P.F., Dye Laser Principles, Elsevier Science (2012).

3. El-Kashef, H. and Hassan, G.E. "Studies of problems connected with wide scan dye laser spectroscopy", Opt. Mater., 14(1), pp. 19-24 (2000).

4. Duarte, F.J., Tunable Laser Applications, CRC Press (2010).

5. Gurian, J.H., Maeda, H. and Gallagher, T.F. "Kilohertz dye laser system for high resolution laser spectroscopy", Rev. Sci. Instrum., 81(7), p. 073111 (2010).

6. Schumacher, D., Marshall, O., Holt, J., Bajema, M.L., Leeuwen, R.V. and Gallagher, T.F. "kHz dye laser for use with ultrafast laser systems", Appl. Opt., 41(9), pp. 1722-1724 (2002).

7. Jelínková, H., Lasers for Medical Applications: Diagnostics, Therapy and Surgery, 1st Edn, Elsevier (2013).

8. Yin, N., Choudhary, S. and Nouri, K. "Pulsed dye laser for the treatment of nail psoriasis", Cutis., 92(3), pp. 129-135 (2013).

9. Kashlan, L., Graber, E.M. and Arndt, K.A. "Hair dryer use to optimize pulsed dye laser treatment in Rosacea patients", J. Clin. Aesthet. Dermatol., 5(6), p. 41 (2012).

10. McKenzie, A.L. and Carruth, J. "A comparison of gold-vapour and dye lasers for photodynamic therapy", Lasers. Med. Sci., 1(2), pp. 117-120 (1986).

11. Lee, S.J., Choi, M.J., Zheng, Z., Chung, W.S., Kim, Y.K. and Cho, S.Bin. "Combination of 595-nm pulsed dye laser, long-pulsed 755-nm alexandrite laser, and microdermabrasion treatment for keratosis pilaris: retrospective analysis of 26 Korean patients", J. Cosmet. Laser. Ther., 15(3), pp. 150-154 (2013).

12. Yan, Z., Hu, X., Guo, S. and Cheng, Y. "Dye lasers used in CSSAR sodium fluorescence Doppler lidar", International Symposium on Photoelectronic Detection and Imaging, 8192, p. 819240 (2011).

13. Hake, R., Arnold, D.E., Jackson, D.W., Evans, W.E., Ficklin, B.P. and Long, R.A. "Dye-laser observations of the nighttime atomic sodium layer", J. Geophys. Res., 77(34), pp. 6839-6848 (1972).

14. Jones, A.L., DeYoung, R.J. and Elsayed-Ali, H.E. "Compact solid-state dye polymer laser for ozone lidar applications", Opt. Eng., 41(11), pp. 2951-2958 (2002).

15. Duarte, F.J., High Power Dye Lasers, 1st Edn., Springer-Verlag Berlin Heidelberg (2012).

16. Sahyun, M.R.V. and Sharma, D.K. "Photophysics of rigidized 7-aminocoumarin laser dyes: efficient intersystem crossing from higher excited state", Chem. Phys. Lett., 189(6), pp. 571-576 (1999).

17. Pavlopoulos, T.G. "Scaling of dye lasers with improved laser dyes", Prog. Quant. Electron., 26(4), pp. 193-224 (2002).

18. Redmond, R.W., Kochevar, I.E., Krieg, M., Smith, G. and McGimpsey, W.G. "Excited state relaxation in cyanine dyes: a remarkably efficient reverse intersystem crossing from upper triplet levels", J. Phys. Chem. A, 101(15), pp. 2773-2777 (1997).

19. Singh, N., Patel, H.K., Dixit, S.K. and Vora, H.S. "Design, modeling, and performance evaluation of a novel dye cell for a high repetition rate dye laser", Rev. Sci. Instrum., 83(10), p. 105114 (2012).

20. Link, S. and El-Sayed, M.A. "Size and temperature dependence of the plasmon absorption of colloidal gold nanoparticles", J. Phys. Chem. B., 103(21), pp. 42124217 (1999).

21. Evanoff, D.D. and Chumanov, G. "Size-controlled synthesis of nanoparticles. 2. Measurement of extinction, scattering, and absorption cross sections", J. Phys. Chem. B., 108(37), pp. 13957-13962 (2004).

22. Sun, Y. and Xia, Y. "Shape-controlled synthesis of gold and silver nanoparticles", Science., 298(5601), pp. 2176-2179 (2002).

23. Lang, X., Qian, L., Guan, P., Zi, J. and Chen, M. "Localized surface plasmon resonance of nanoporous gold", App. Phys. Lett., 98(9), p. 093701 (2011). 
24. Grabar, K.C., Freeman, R.G., Hommer, M.B. and Natan, M.J. "Preparation and characterization of $\mathrm{Au}$ colloid monolayers", Anal. Chem., 67(4), pp. 735-743 (1995).

25. Li, H. and Rothberg, L. "Colorimetric detection of DNA sequences based on electrostatic interactions with unmodified gold nanoparticles", Proc. Natl. Acad. Sci. U.S.A., 101(39), pp. 14036-14039 (2004).

26. Mohammadi, A. Sandoghdar, V. and Agio, M. "Gold nanorods and nanospheroids for enhancing spontaneous emission", New J. Phys., 10(10), p. 105015 (2008).

27. Geddes, C.D. and Lakowicz, J.R. "Metal-enhanced fluorescence", J. Fluoresc., 12(2), pp. 121-129 (2002).

28. Dong, L., Ye, F., Chughtai, A., Liuolia, V., Popov, S., Friberg, A.T. and Muhammed, M. "Lasing from water solution of Rhodamine $6 \mathrm{G}$ /gold nanoparticles: impact of-coating on metal surface", IEEE J. Quant. Electron., 48(9), pp. 1220-1226 (2012).

29. Kumar, B.R., Basheer, N.S., Kurian, A. and George, S.D. "Thermal-Lens study on the distance-dependent energy transfer from Rhodamine $6 \mathrm{G}$ to gold nanoparticles", Int. J. Thermophys., 34(10), pp. 11982-1992 (2013).

30. Kumar, B.R., Basheer, N.S., Kurian, A. and George, S.D. "Study of concentration-dependent quantum yield of Rhodamine $6 \mathrm{G}$ by gold nanoparticles using thermal-lens technique", App. Phys. B., 115(3), pp. 335-342 (2014).

31. Khatua, S., Ribeiro P., Pedro, M., Yuan, H., Gupta, A., Zijlstra, P. and Orrit, M. "Resonant plasmonic enhancement of single-molecule fluorescence by individual gold nanorods", Acs. Nano., 8(5), pp. 4440-4449 (2014).

32. Yorulmaz, M., Khatua, S., Zijlstra, P., Gaiduk, A. and Orrit, M. "Luminescence quantum yield of single gold nanorods", Nano. Lett., 12(8), pp. 4385-4391 (2012).

33. Mertens, H., Koenderink, A.F. and Polman, A. "Plasmon-enhanced luminescence near noble-metal nanospheres: comparison of exact theory and an improved Gersten and Nitzan model", Phys. Rev. B., 76(11), p. 115123 (2007).

34. Lee, K.G., Eghlidi, H., Chen, X.W., Renn, A., Götzinger, S. and Sandoghdar, V. "Spontaneous emission enhancement of a single molecule by a doublesphere nanoantenna across an interface", Opt. Express., 20(21), pp. 23331-23338 (2012).

35. Anger, P., Bharadwaj, P. and Novotny, L. "Enhancement and quenching of single-molecule fluorescence", Phys. Rev. Lett, 96(11), p. 113002 (2006).

36. Mohammadi, A., Sandoghdar, V. and Agio, M. "Gold, copper, silver and aluminum nanoantennas to enhance spontaneous emission", J. Comput. Theor. Nanosci., 6(9), pp. 2024-2030 (2009).

37. Mertens, H. and Polman, A. "Strong luminescence quantum-efficiency enhancement near prolate metal nanoparticles: Dipolar versus higher-order modes", $J$. App. Phys., 105(4), p. 044302 (2009).
38. Sen, T., Sadhu, S. and Patra, A. "Surface energy transfer from rhodamine $6 \mathrm{G}$ to gold nanoparticles: A spectroscopic ruler", App. Phys. Lett., 91(4), p. 043104 (2009).

39. Sen, T. and Patra, A. "Resonance energy transfer from Rhodamine $6 \mathrm{G}$ to gold nanoparticles by steady-state and time-resolved spectroscopy", J. Phys. Chem. C., 112(9), pp. 3216-3222 (2008).

40. Haldar, K.K., Sen, T. and Patra, A. “Au@ ZnO coreshell nanoparticles are efficient energy acceptors with organic dye donors", J. Phys. Chem. C., 112(31), pp. 11650-11656 (2008).

41. Dulkeith, E., Ringler, M., Klar, T.A., Feldmann, J., Munoz, J.A. and Parak, W.J. "Gold nanoparticles quench fluorescence by phase induced radiative rate suppression", Nano. Lett., 5(4), pp. 585-589 (2005).

42. Dulkeith, E., Morteani, A.C., Niedereichholz, T., Klar, T.A., Feldmann, J., Levi, S.A., Van Veggel, F., Reinhoudt, D.N., Möller, M. and Gittins, D.I. "Fluorescence quenching of dye molecules near gold nanoparticles: radiative and nonradiative effects", Phys. Rev. Lett., 89(20), p. 203002 (2002).

43. Yuan, H., Khatua, S., Zijlstra, P., Yorulmaz, M. and Orrit, M. "Thousand-fold enhancement of singlemolecule fluorescence near a single gold nanorod", Angew. Chem. Int. Ed., 52(4), pp. 1217-1221 (2013).

44. Ayala Orozco, C., Liu, J.G., Knight, M.W., Wang, Y., Day, J., Nordlander, P. and Halas, N.J. "Fluorescence enhancement of molecules inside a gold nanomatryoshka", Nano. Lett., 14(5), pp. 2926-2933 (2014).

45. Mohammadi, A., Kaminski, F., Sandoghdar, V. and Agio, M. "Fluorescence enhancement with the optical (Bi-) conical antenna", J. Phys. Chem. C., 114(16), pp. $7372-7377$ (2010).

46. Noginov, M.A., Zhu, G., Bahoura, M., Small, C.E., Davison, C., Adegoke, J., Drachev, V.P., Nyga, P. and Shalaev, V.M. "Enhancement of spontaneous and stimulated emission of a rhodamine $6 \mathrm{G}$ dye by an $\mathrm{Ag}$ aggregate", Phys. Rev. B., 74(188), pp. 184203 (2006).

47. Cushing, S.K., Li, J., Bright, J., Yost, B.T., Zheng, P., Bristow, A.D. and Wu, N. "Controlling plasmoninduced resonance energy transfer and hot electron injection processes in Metal@ TiO2 core-shell nanoparticles", J. Phys. Chem. C., 119(28), pp. 16239-16244 (2015).

48. Spinelli, P. and Polman, A. "Prospects of near-field plasmonic absorption enhancement in semiconductor materials using embedded Ag nanoparticles", Opt. Express., 20(105), pp. A641-A654 (2012).

49. Liaw, J.W., Chen, J.H., Chen, C.S. and Kuo, M.K. "Purcell effect of nanoshell dimer on single molecules fluorescence", Opt. Express., 17(16), pp. 13532-13540 (2009). 


\section{Biography}

Ahmad Moshaii, Associate Professor, received his PhD degree from Physics Department of Sharif University of Technology in 2004 in the field of sonoluminescence. Then he worked at IPM as a Postdoc researcher with concentration on RPC detectors. Since
May 2008, Dr. Moshaii has been working with Physics Department of Tarbiat Modares University (TMU); he has established a nanooptics lab there. Currently, his research interest concentrates on plasmonic and solar harvesting materials, including perovskite solar cells and photoelectrochemical water splitting cells. 